\title{
Comparison between Radiological and Invasive Diagnostic Modalities in Diagnosis of Breast Cancer
}

\author{
Gulcin Ozkan Onur ${ }^{1 *}$, Ercument Tarcan², Asim Onur ${ }^{3}$, Huseyin Can ${ }^{4}$, Murat \\ Kemal Atahan ${ }^{2}$, Seyran Ceri Yigit ${ }^{5}$, Fulya Cakalagaoglu ${ }^{5}$
}

\begin{abstract}
Background: Breast cancer is the most common cause of deaths of cancer in women. Nowadays, following completion of imaging methods, mainly fine needle aspiration biopsy (FNAB) and core biopsy methods have been used for establishing cytopathological diagnosis although discussions regarding superiority continue. Materials and Methods: Those with a complaint of "mass in breast" along with those diagnosed to have a mass as a result of routine physical examination among all patients applying to our clinic between 01.01 .2009 and 31.12.2011 were retrospectively assessed. Totals of 146 and 64 patients with complete radiological observation who had undergone FNAB and core biopsies, respectively, were evaluated. Postoperative pathological results of patients of both groups receiving surgery were also taken into consideration. All results were compared in terms of false positivity/negativity, sensitivity/specifity, surgery types and distribution of postoperative results with regard to diagnoses along with those of malignant/benign masses with regard to quadrants determined. Results: Diagnostic malignancy power of mammographic BIRADS classification was 87.3\%. However, the value was $75 \%$ in the core biopsy group. Sensitivity and specifity following comparison of FNAB and postoperative pathology results of those receiving surgery were $85.4 \%$ and $92.9 \%$ while they were $93.5 \%$ and $100 \%$ in the core biopsy group. Diagnostic malignancy power, calculated by determining AUC in ROC analysis, of FNAB was 89.1\% while that of core biopsy was $96.7 \%$. Conclusions: It was shown that core biopsy is superior to FNAB in terms of sensitivity, specificity and accurate histopathological classification. However; quick, cheap and basic diagnosis by means of FNAB should not be ignored. Sensitivity of FNAB is rather high in experienced hands and furthermore it would be expected to be lower than with core biopsy.
\end{abstract}

Keywords: Breast cancer - FNAB - core biopsy - sensitivity - specificity - comparison

Asian Pac J Cancer Prev, 16 (10), 4323-4328

\section{Introduction}

The most common cause of deaths due to cancer amongst women is breast cancer. Any mass defined in the physical examination of a woman over her 40 age must be considered as a potential cancer unless it is disproved and from that point every diagnostic test must be analyzed to differentially diagnose the cancer option. Mean while the fact as $30 \%$ of the breast cancers have been obtained under the age of 50 must not be omitted (Kumar et al., 2009; Jemal et al., 2011).

The approach to the mass in breast must be started by physical examination followed by radiological imaging and then if necessary biopsy analysis must be performed. In the evaluation of breast lesions fine needle aspiration (FNA) and core biopsy have been commonly applied methods. Utilization of core biopsy in the breast cancer diagnosis have been induced since there have been an implication about the topic in scientific literature. However the situation of core biopsy in the utility of diagnosing breast cancer has been compared to the place of FNA only in a few researches. Likewise it has been still on a debate that which one of the methods must be placed in routine application (Shannon et al., 2001; Lieske et al., 2006; Willems et al., 2012, Farshid et al., 2014).

The purpose of this research has been the comparative analysis of the results obtained by the techniques such as radiological viewing, fine needle aspiration biopsy and core biopsy in breast tumors determined by physical examination or radiological imaging. Also it has been aimed to compare the sensitivity /specificity and false positivity/negativity of these techniques by controlling the

${ }^{1}$ Karakavak Family Medicine Center, Malatya, ${ }^{2}$ Izmir Katip Celebi University Atatürk Education and Research Hospital, General Surgery Clinic, Izmir, ${ }^{3}$ Department of General Surgery, Medical Faculty, Inonu University Malatya, ${ }^{4}$ Family Medicine Clinic, ${ }^{5}$ Pathology Clinic, Izmir Katip Celebi University Atatürk Education and Research Hospital, Izmir, Turkey *For correspondence: gulcinozkann@yahoo.com 
postoperative pathological results. This research aims to inform the clinicians about which method has the priority in diagnosis of breast cancer.

\section{Materials and Methods}

The data of the women who made an application to Izmir Ataturk Education and Research Hospital General Surgery Clinic in between 01/01/2009-31/12/2011 dates with complaints of breast mass or determined breast mass during her routine examination have been retrospectively approached. These women have been applied with FNAB and/or core biopsy and radiological examination (ultrasound and mammography). Male patients have been omitted. Patients have been defined into two groups; FNAB applied patients $(n=146)$ and core biopsy applied patients $(n=64)$.

In core biopsy analysis; samples have been obtained by needle suitable for the length and the width of the lesions utilizing the Bord max core biopsy machine. They have been fixed in $10 \%$ formaldehyde in pathology laboratory before being tracked overnight as a routine in a fully automatic tissue tracking device. After samples were embedded into paraffin they have been sliced in the width of 4-5 micron in rotary microtome. HematoxylinEosin staining has been applied on the samples and these core biopsy materials have been evaluated in pathology laboratory of the hospital.

In fine needle aspiration biopsy; $10 \mathrm{cc}$, needle diameter 0,7 mm, 23-25 gauge injectors have been used to increase the negative pressure. Samples have been sprayed on to the 4-6 lames by the help of handler. Half of the lames have been left to be dried with air for an hour before stained with giemsa. Other lames have been fixed in $96 \%$ ethanol for 30 minutes and then stained with papanicolaou stain. All the lames with FNAB have been evaluated in pathology laboratory of the hospital under the routine light microscopy.

Patients with benign lesions in addition to the patients taken to additional procedures have been traced. Some of the patients with malign results have been directed to neoadjuvant therapy. And some of those have been operated after the completion of the arrangements. The pathological results of specimens from the operated patients in both groups have been included in this research. All results have been compared in the aspect of false positivity or negativity, specificity and sensitivity. The results also have been defined in a dial plate of benign and malign lesions due to their distributions among the operational types, post operative pathology result diagnosis.

Statistical analysis of the data has been held by SPSS 15.0 for Windows and MedCalc demo package with 95\% trust. Numbers, percentages, standard mean and standard deviations (minimum and maximum values) have been used in defining values. For the adaption of Radiological BIRADS results of the cases with pathology results Kappa adaption and ROC curve analysis have been used. ROC curves have been drawn with MedCalc demo program. $P$ value under 0.05 has been taken to be statistically significant.

\section{Results}

Totally 210 biopsy results have been included in this research (64 core biopsies and 146 FNAB). The average age of the patients with core biopsy has been $47 \pm 14$. 16 (29-88) year; FNAB applied group have been 50.21 \pm 13.06 $(18-88)$ years old.

$84(57.5 \%)$ of the FNAB have been obtained from left and 61(41.8\%) of it have been obtained from right breast. Only one lesion has been defined to be in bilateral localization. Most of the lesions have been in the upperouter localization $(n=82) 56.2 \%$. Some have been in the upper inner; $(n=14) 9.6 \%$. Lesions in the lower outer localization have had $(n=16) 11.0 \%$. Lower inner localized lesions have been $n=8,5.5 \%$. Lesions in the retroareolar area have been $(n=8) 5.5 \%$. Lesions in the subareolar area have been $(n=7) 4.8 \%$; periareolar area $(n=1) 0.7 \%$; axillary tail $(n=1) 0.7 \%$; at the 12 hour side $(n=7) 4.8 \%$; covering the whole breast $(n=1) 0.7 \%$; covering the upper half of the breast $(n=1) 0.7 \%$.

$35(54.7 \%)$ of the core biopsies have been applied to the left breast. 29 (45.3\%) of the core biopsies have been applied to right breast. Lesions have been detected to be mostly on the outer quadrants (upper out quadrant: $n=4$ $6.3 \%$; lower inner quadrant: $n=1,1.6 \%$; retroareolar area: $\mathrm{n}=3,4.7 \%$; axillary area: $\mathrm{n}=1,1.6 \%$; multicentre: $\mathrm{n}=1$, $1.6 \%$; periareolar area: $n=1,1.6 \%$ ). Also lesions have been detected to be mostly at upper outer quadrant (upper outer quadrant: $n=50,78.1 \%$; upper inner quadrant: $n=3,4.7 \%$ ).

83 of the patients with fine needle aspiration application have been operated. Both malign and benign tumors have been located mostly at upper outer quadrant. 49 of the patient with core biopsy have been operated. Malign lesions have been mostly detected in the upper

Table 1. FNAB Results According to Radiologic Results of the FNAB Applied Group

\begin{tabular}{|c|c|c|c|c|c|c|c|}
\hline & & \multicolumn{4}{|c|}{ PATHOLOGY RESULTS } & \multicolumn{2}{|c|}{ Total } \\
\hline & & \multicolumn{2}{|c|}{ Malign } & \multicolumn{2}{|c|}{ Benign } & \multirow[b]{2}{*}{$\mathrm{n}$} & \multirow[b]{2}{*}{$\%$} \\
\hline & & $\mathrm{n}$ & $\%$ & $\mathrm{n}$ & $\%$ & & \\
\hline \multicolumn{8}{|c|}{ Fine needle aspiration biopsy applied group } \\
\hline \multirow[t]{3}{*}{ RADIOLOGY BIRADS } & Malign & 37 & 94.9 & 17 & 20.2 & 54 & 43.9 \\
\hline & Benign & 2 & 5.1 & 67 & 79.8 & 69 & 56.1 \\
\hline & Total & 39 & 31.7 & 84 & 68.3 & 123 & 100.0 \\
\hline \multicolumn{8}{|l|}{ Core biopsy applied group } \\
\hline \multirow[t]{3}{*}{ RADIOLOGY BIRADS } & Malign & 50 & 100.0 & 6 & 50.0 & 56 & 90.3 \\
\hline & Benign & 0 & 0.0 & 6 & 50.0 & 6 & 9.7 \\
\hline & Total & 50 & 80.6 & 12 & 19.4 & 62 & 100.0 \\
\hline
\end{tabular}


outer quadrant with $83.3 \%$. Only one benign lesion has been located in the axillary area.

Diagnostic distributions have been investigated according to postoperative pathology results. Invasive ductal carcinoma amongst all diagnosis of the FNAB applied group has been the most common with $36.1 \%$ ratio. (Fibroadenoma $15.7 \%$, fibrocystic changes $13.3 \%$ ). The only benign lesion obtained in the core biopsy applied groups has been diagnosed to be fibroadenoma. Invasive ductal carcinoma has been found to be the most common lesion amongst the malign diagnosis $62.5 \%$.

Kappa compliance analysis has been utilized for the FNAB results and the radiology results. The kappa coefficient has been 0.677 with a p value equal to 0.001 . The compliance between the two techniques have been revealed to be significantly enough $(\mathrm{p}<0.05)$. Also for the comparison of core biopsy results and radiology results kappa compliance analysis has been used the kappa coefficient have been 0.617 with a p value equal to 0.001 . The compliance between the two techniques have been revealed to be significantly enough $(\mathrm{p}<0.05)$ (Table 1$)$.

Diagnostic efficiency of the radiology results with respect to pathology results have been investigated with ROC analysis. AUC (Area Under Curve) for the FNAB group have been calculated to be 0.873 ( $p=0.001)$. Reliability of the radiology BIRADS classification for the malignity have been calculated to be $87.3 \%(\mathrm{p}<0.05)$ (Graph 1/a). AUC for the core biopsy results have been calculated as $0.750(p=0,001)$. Reliability of the radiology BIRADS classification for the malignity have been calculated to be $75.0 \%(\mathrm{p}<0.05)($ Graph $1 / \mathrm{b})$

Sensitivity of FNAB results in the BIRADS classification (mammography) with respect to radiology results have been obtained as $94.9 \%$ and the specifity have been $79.8 \%$. Specificity and sensitivity of the core biopsy results in BIRADS classification with respect to radiology results have been found to be $100 \%$ and $50 \%$ respectively (Table 2).

When the radiology results of FNAB cases have been compared to postoperative pathology results, $45(97.8 \%)$ cases out of $55(66.3 \%)$ cases assigned to be malign in the radiology have been observed to be malign indeed in the postoperative pathology results. 10 cases $(27.0 \%)$ have been diagnosed to be benign in the pathology results. $27(73.0 \%)$ cases out of the $28(33.7 \%)$ cases assigned to be benign according to the pathology results have been observed to be benign indeed in the postoperative pathology results. Only one case $(2.2 \%)$ has been diagnosed to be malign lesion. The kappa coefficient have been calculated as $0.725(\mathrm{p}=0.001)$ in the kappa compliance analysis of radiology and postoperative pathology results. The compliance has been found to be enough significantly between the two tests $(\mathrm{p}<0.05)$. Also ROC analysis has been used to test the efficiency of the radiology results with respect to postoperative pathology results; AUC $(95 \% \mathrm{Cl})$ have been calculated as 0.854 $(0.759-0.922)(\mathrm{p}=0.001)$. The sufficiency of radiology in diagnosis for the BIRADS malignity classification has been assigned to be $85.4 \%(\mathrm{p}<0.05)$. According to radiology results of the FNAB applied cases the specificity and the sensitivity for the post operational pathology results for the BIRADS classification have been $97.8 \%$ and 73.0\% respectively (PPV:81.8; NPV:96.4; PPR:3.62; NPR:0.03).

Malignity has been suggested in the radiology comments of all of the operated core biopsy applied patients. Only one of those patients has been diagnosed with a benign lesion according to the postoperative pathology results. Other 48 patients have been diagnosed to have a malign lesion according to the postoperative pathology results which have been harmonious with the radiology comments. Kappa and ROC analysis have not been applied to those patients since all of the core biopsy applied patients with the postoperative pathology results have been found to have malign lesions according to the BIRADS classification and no false or true negative results have been observed.

In the kappa compliance analysis applied for the pre and postoperative results of the FNAB applied patients (FNAB results of 14 patients have been non-diagnostic and they have been omitted), the kappa coefficient has been calculated as $0.765(\mathrm{p}=0.001)$. The compliance between both techniques have been found to be significantly sufficient in statistics $(\mathrm{p}<0.05)$. In the kappa compliance analysis for the core biopsy results and post operative pathology results of patients (two of the cases have been

Table 2. Diagnostic Test Results of FNAB and Core Biopsy according to Radiology Results

\begin{tabular}{lcc}
\hline Diagnostic test & $\begin{array}{c}\text { FNAB } \\
\text { group } \\
\text { Preoperational } \\
\text { Pathology }\end{array}$ & $\begin{array}{c}\text { Core Biopsy } \\
\text { group } \\
\text { Preoperational } \\
\text { Pathology }\end{array}$ \\
\hline Sensitivity & 94.9 & 100 \\
Specificity & 79.8 & 50 \\
Positive Predictive Value(PPV) & 68.5 & 89.3 \\
Negative Predictive Value(NPV) & 97.1 & 100 \\
Positive Predictivity Ratio(PPR) & 4.69 & 2 \\
Negative Predictivity Ratio(NPR) & 0.06 & 0 \\
& $\mathrm{n}$ & $\mathrm{n}$ \\
True positive & 37 & 50 \\
False positive & 17 & 6 \\
True negative & 67 & 6 \\
False negative & 2 & 0 \\
AUC (95\% CI) & ROC analysis \\
& 0.873 & 0.750 \\
AUC p value & $(0.801-0.926)$ & $(0.624-0.851)$ \\
\hline & 0.001 & 0.001 \\
\hline
\end{tabular}

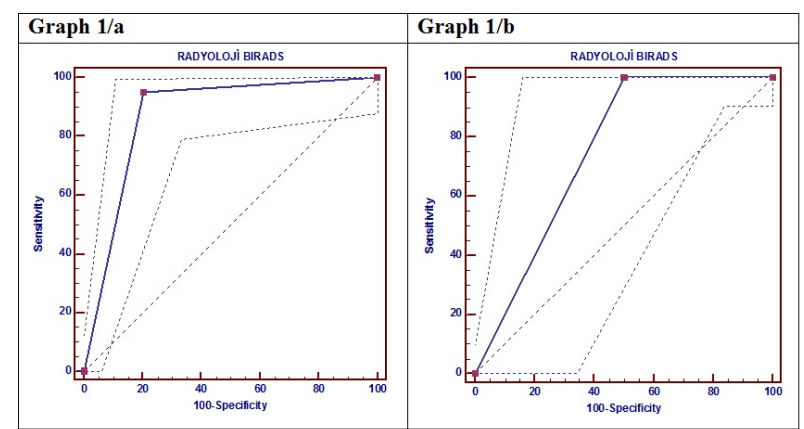

Figure 1. ROC Curve Drawn for the Comparison of Radiology Results with Respect to Core and Fine Needle Aspiration Biopsy Pathology Results 
omitted since the breast tissue have not been obtained in the sample) the kappa coefficient has been calculated as 0.379 ( $\mathrm{p}=0.001)$. The compliance between both techniques have been found to be significantly sufficient in statistics $(\mathrm{p}<0.05)$

ROC analysis has been held for the investigation of diagnostic sufficiency of FNAB with respect to post operative pathology results. AUC have been calculated as $0.891(p=0.001)$. Efficiency of fine needle aspiration biopsy in the malignity diagnosis have been found to be $89.1 \%(\mathrm{p}<0.05)$. Also ROC analysis has been held for the investigation of diagnostic sufficiency of core biopsy with respect to postoperative pathology results. AUC has been calculated as 0.967 ( $\mathrm{p}=0.113$ ). Efficiency of core biopsy in the diagnosis of malignity has been found to be $96.7 \%$. But since the $\mathrm{p}$ value has been calculates to be over 0.05 these results has been assigned to be not significant.

Sensitivity and specificity of the preoperative pathology results in FNAB applied cases with respect to postoperative pathology results have been calculated as $85.4 \%$ and $92.9 \%$ respectively. Sensitivity and specificity of the preoperative pathology results in core biopsy applied cases with respect to postoperative pathology results have been calculated as $93.5 \%$ and $100 \%$ respectively.

\section{Discussion}

Breast cancer has been recently raised. This has been followed by the technologic improvement in the imaging systems and the screening methods. As a result breast lesions to be diagnosed and biopsy numbers have been increased. Currently the techniques utilized for the purpose have been most commonly FNAB and core biopsy (Aksaz et al., 2005).

FNAB have been applied in many centers for the preoperative diagnosis of palpable breast lesions for a long time. It has been revealed as a simple, cheap, quick and partially less painful method. The sensitivity of FNAB in the scientific literature has been calculated as $35-95 \%$, and the specificity has been calculated as $48-100 \%$ in different researches (Willems et al., 2012). Also the specificity and sensitivity of FNAB in the metaanalytic researches have been calculated as 76-100\% and 60-100\% respectively (Chaiwun and Thorner, 2007; Akçil et al., 2008). In our research FNAB results compared to postoperative pathology results has been obtained to be in a compliance with the scientific literature in the aspect of specificity and sensitivity. Positive predictive value can be defined as the possibility of the tested patients with positive results to be sick in reality. It depends on the prevalence and specificity. High positive predictive value reduces the risk of unnecessary therapy. Negative predictive value can be defined as the possibility of the tested patients not to be sick in reality. High negative predictive value diminishes the risk of unnecessary therapy. In research with a wide range of cases (Boerner et al., 1999), positive predictive value (PPV) has been calculated as $99.3 \%$, negative predictive value (NPV) has been calculated as $96.2 \%$. In this research PPV has been calculated as $94.6 \%$, NPV has been calculated as $81.2 \%$. When non-diagnostic results have been omitted truth ratio of FNAB has raised in a false way. 23 (15\%) results out of the 146 FNAB results have been obtained as non-significant in this study. Values in between 4-17\% have been presented in the previous studies. In 2008 in research of Barra et al amongst 264 patients this ratio were found to be $14 \%$ and was acceptable since it was under the $20 \%$ limit. But it was still over the $10 \%$ ratio reported by the experts (Chaiwun et al., 2002; Barra et al., 2008). The sensitivity and specificity have been calculated as $57 \%$ and $96 \%$ respectively by Lifrange et al (omitting the non-diagnostic cases). Ibrahim et al suggested in their research that the sensitivity and specificity could have been raised up to $100 \%$ when the non-diagnostic cases have been omitted (Lifrange et al., 1997; Ibrahim et al., 2001).

FNAB have been a commonly used method for the diagnosis of palpable lesions. Advantages of FNAB have been its low cost, quick resulting, low complication risk and comparable results with respect to core biopsy (Ariga et al., 2002; Abdel-Hadi et al., 2010; Rosa et al., 2012). It has been more suitable in the patients with a lesion very close to the skin or the patients using anticoagulation therapy. But it depends on the experience and ability of FNAB cytopathologists. (Gornstein et al., 2004; Lieske et al., 2006; Willems et al., 2012). Also FNAB has been not possible to utilize for the differentiation of in situ cancers and invasive cancers. Furthermore FNAB have been found to be insufficient for the cytological grading of the malign cases (Shin and Sniege, 1998; Nerurkar and Osin, 2003). Average success ratio for the FNAB in palpable lesions has been defined in between 75-90\%, in non-palpable lesions between $34-58 \%$. Whereas the same ratios for the core biopsy have been obtained as $97 \%$ and $94 \%$ respectively (Pisano et al., 1998; Leifland et al., 2003; Willems et al., 2012). This suggests the higher efficiency of core biopsy in the diagnosis of non-palpable lesions in the aspect of higher sensitivity and specificity (Agacayak et al., 2014).

In the subscribed researches FNAB has been demonstrated to have more specific and sensitive on the diagnosis of benign and malign lesions (Litherland et al., 1996; Ibrahim et al., 2001)). In scientific literature core biopsy has been demonstrated to have specificity between 85-100\% and sensitivity between 93-100\%. In the research made by Caruso et al in 1998 core biopsy was shown to have a sensitivity ratio as $92 \%$ and specificity ratio as $100 \%$. Also positive predictive value has been detected as $100 \%$ (Caruso et al., 1998). In our project the comparison of core biopsy results with the postoperative pathology results have been demonstrated that core biopsy results had a sensitivity ratio as $93.5 \%$, specificity ratio as $100 \%$ and positive predictive value have been calculate as $100 \%$. Again in the research of Caruso et al no false positive results have been obtained whereas six false negative results have been detected due to sampling mistakes. Two of those have been related to the small size of the tumor and three of those have been related to well known phenomena of mixture in the dysplastic and neoplastic formations in the breast cancer. For the last case it has been related to quite deep localization of lesion. (Caruso et al., 1998). In our research three false negative results have been obtained. In one case the result of the core biopsy applied to the axillary lymphadenopathy has been shown 
to be epithelial tumor metastasis, so the patient has been operated. On the other two cases since the location of the lesion and the size of the lesion have been found not to be suitable sample could not be obtained. Since the radiology comments have been in the malignity direction these patients have been recommended to repeat the biopsy. In our study four cases have been defined to be benign by the core biopsy results and the three of them have been demonstrated to be malign lesions by the post operative pathology so the power of core biopsy in diagnosing the malignity has been calculated as $96.7 \%$. Unfortunately the results have been found to be statistically no significant.

The utilization of small gauge needle during the core biopsy procedure diminishes the trauma depended complications, pain and the risk of tumor implanting to the biopsy area (Nerurkar and Osin, 2003). Some of the most important advantages obtained by the core biopsy in the diagnosis of breast cancer have been that estrogens and progesterone receptors could have been detected and c-erb B2 analysis could be held. These properties have a crucial role for the neoadjuvant therapy of the advanced stage cases. In the scientific literature core biopsy has been reported to detect such cases in a great success (MuellerHolzner et al., 2001; Shousha 2003). Also in FNAB group the differentiation of in situ and invasive cancer can not been achieved. Whereas in core biopsy the differentiation between in situ and invasive cancer can be achieved in a defined confidence range. In scientific literature the diagnosing DCIS by core biopsy have been demonstrated to be in the range of $35-82 \%$ (Litherland et al., 1996). In the research of Michael S.P. Cheng et al (2003) 148 patients with DCIS diagnosis have been followed for four years. 63 of them have been applied core biopsy and the certain DCIS diagnosis has been assigned to 35 of them (Michael et al., 2003). Also in a similar research of Frayne et al (1996) DCIS diagnosis by core biopsy have been reached to the ratio of $56 \%$. However in both studies when atypical and suspicious cases have been included these ratios raise to $90 \%$ and $78 \%$ respectively (Frayne et al., 1996). In our research one patient have been diagnosed false negatively as low grade ductal epithelial hyperplasia but the result has been obtained as ductal carcinoma in situ by the postoperative pathology analysis.

Another advantage of the core biopsy is that it is less dependent on the quality of the experience and the skills of the cytopathologists than FNAB. Furthermore in the diagnosis of microcalcifications which have not been included in this research core biopsy has a crucial role and the material obtained has a chance to be analyzed in radiological imaging (Thom et al., 2009).

In our research the diagnostic efficiency of mammography in the malignity amongst the FNAB group have been calculated as $87.3 \%$ and amongst the core biopsy group it has been calculated as $75 \%$ with respect to evaluation of the radiology. In the prospective research held by Clarke et al (2001) comments of radiology on cancer diagnosis with respect to cytological analysis calculated to have $61 \%$ sensitivity, with respect to core biopsy results calculated as $97 \%$ sensitivity. When the radiology comments have not been on the cancer direction its sensitivity with respect to cytology have been calculated as $53 \%$ and with respect to core biopsy it has been calculated as $95 \%$. This suggests the superiority of core biopsy over the FNAB.

As a result in this research the superiority of the core biopsy has been demonstrated in the aspect of specificity, sensitivity and true histopathological classification. On the other hand the advantage of FNAB in the aspect of quick results, low costs, and basic diagnosis cannot be omitted. In the hands of expert specialists the sensitivity of FNAB have been found to be high but still found to be lower values than core biopsy results. Especially security of core biopsy in the application of non-palpable, small, deeply localized lesions and its higher sensitivity for the diagnosis of atypical and fibroepithelial lesions suggests superiority of the technique over FNAB. In addition to that core biopsy can provide the information of receptor condition, proliferative activity, pyloidy, c-erb B and p53 oncogene and tumor suppressor expressions in preoperative interval. These parameters have been found to be necessary for the operational or neoadjuvant therapy. So this also suggests the advantages and superiority of the core biopsy over FNAB.

\section{References}

Abdel-Hadi M, Abdel-Hamid GF, Abdel-Razek N, Fawzy RK (2010). Should fine-needle aspiration cytology be the first choice diagnostic modality for assessment of all nonpalpable breast lesions? The experience of a breast cancer screening center in Alexandria, Egypt. Diagn Cytopathol, 38, 880-9.

Agacayak F, Ozturk A, Bozdogan A, et al (2014). Stereotactic vacuum-assisted core biopsy results for non-palpable Breast Lesions. Asian Pac J Cancer Prev, 15, 5171-4.

Akçil M, Karaagaoglu E, Demirhan B (2008). Diagnostic accuracy of fine needle aspiration cytology of palpabl breast masses. An SROC curve with fixed and random effects linear meta-regression models. Diagn Cytopathol, 36, 303-10.

Aksaz E, Öncel T, Atasoy G, et al (2005). Kor biyopsi açık biyopsilerin yerini alabilir mi? (Turkish) $J$ Breast Health, 1, 18-21.

Ariga R, Bloom K, Reddy VB, et al (2002). Fine-needle aspiration of clinically suspicious palpabl breast masses with histopathologic correlation. Am J Surg, 184, 410-3.

Barra A, Gobbi H, Rezende CA, et al (2008). Comparison of aspiration cytology and core needle biopsy according to tumor size of suspicious breast lesions. Diagn Cytopathol, 36, 26-31.

Boerner S, Fornage BD, Singletary E, et al (1999). Ultrasound guided fine-needle aspiration (FNA) of nonpalpabl breast lesions: a review of 1885 FNA cases using the National Cancer Institute-supported recommendations on the uniform approach to breast FNA. Cancer, 87, 19-24.

Caruso ML, Gabrieli G, Marzullo G, et al (1998). Core biopsy as alternative to fine-needle aspiration biopsy in diagnosis of breast tumors. The Oncologist, 3, 45-9.

Chaiwun B, Settakorn J, Ya-In C, et al (2002). Effectiveness of fine-needle aspiration cytology of breast: analysis of 2375 cases from Northern Thailand. Diagn Cytopathol, 26, 201-5.

Chaiwun B, Thorner P (2007). Fine needle aspiration for evalution of breast masses. Curr Opin Obstet Gynecol, 19, 48-55.

Farshid G, Sullivan T, Jones T, et al (2014). Performance indices of needle biopsy procedures for the assessment of screen detected abnormalities in services accredited by breast screen Australia. Asian Pacific J Cancer Prev, 15, 10665-73. 
Gulcin Ozkan Onur et al

Frayne J, Sterrett GF, Harvery J et al (1996). Stereotactic 14-gauge core-biopsy of the breast: results from 101 patients. ANZ J Surg, 66, 585-91.

Gornstein B, Jacobs T, Bedard Y, et al (2004). Interobserver agreement of a probabilistic approach to reporting breast fine-needle aspirations on ThinPrep. Diagn Cytopathol, 30, 389-95.

Jemal A, Bray F, Center MM, et al (2011). Global Cancer Statistics. CA Cancer J Clin, 61, 69-90.

Ibrahim AE, Bateman AC, Theaker JM, et al (2001). The role and histological classification of needle core biopsy in comparison with fine needle aspiration cytology in the preoperative assessment of impalpable breast lesions. J Clin Pathol, 54, 121-5.

Kumar (2009). Robbins and Cotran Pathologic Basis of Disease 8th edition, 1074-80.

Shannon J, Douglas-Jones AG, Dallimore NS (2001). Conversion to core biopsy in preoperative diagnosis of breast lesions: is it justified by results? J Clin Pathol, 54, 762-5.

Leifland K, Lagerstedt U, Svane G (2003). Comparison of stereotactic fine needle aspiration cytology and core needle biopsy in 522 non-palpabl breast lesions. Acta Radiol, 44, 387-91.

Lieske B, Ravichandran D, Wright D (2006). Role of fine-needle aspiration cytology and core biopsy in the preoperative diagnosis of screen-detected breast carcinoma. Br J Cancer, 95, 62-6.

Lifrange E, Kridelka F, Colin C (1997). Stereotaxic needle-core biopsy and fine-needle aspiration biopsy in the diagnosis of nonpalpable breast lesions: controversies and future prospects. Eur J Radiol, 24, 39-47.

Litherland JC, Evans AJ, Wilson AR, et al (1996). The impact of core-biopsy on preoperative diagnosis rate of screen detected breast cancers. Cli Radiol, 51, 562-5.

Michael S. P. Cheng, Fox J, Stewart A. Hart (2003). Impact of core biopsy on the management of screen-detected ductal carcinoma in situ of the breast. ANZ J Surg, 73, 404-6.

Mueller-Holzner E, Fink V, Frede T, Marth C (2001). Immunohistochemical determination of Her2 expression in breast cancer from core biopsy specimens: A reliable predictor of Her2 status of the whole tumor. Breast Cancer Res Treat, 69, 13-9.

Nerurkar A, Osin P (2003). The role of new diagnostic techniques. Breast Cancer Res, 5, 305-8.

Pisano ED, Fajardo LL, Tsimikas J, et al (1998). Rate of insufficient samples for fine needle aspiration for nonpalpabl breast lesions in a multicenter clinical trial: the Radiologic Diagnostic Oncology Group 5 Study. The RDOG5 investigators. Cancer, 82, 679-88.

Rosa M, Mohammadi A, Masood S (2012). The value of fine needle aspiration biopsy in the diagnosis and prognostic assesment of palpabl breast lesions. Diagn Cytopathol, 40, 26-34.

Shin H J C, Sniege N (1998). Is a diagnosis of infiltrating versus in situ ductal carcinoma of the breast possible in fine needle aspiration specimens? Cancer Cytopathol, 84, 186-91.

Shousha S (2003). Issues in the interpretation of breast core biopsies. Int J Surg Pathol, 11, 167-76.

Tham T, Iyengar KR, Taib NA, Yip C (2009). Fine needle aspiration biopsy, core needle biopsy or excision biopsy to diagnose breast cancer-Which is the ideal method? Asian Pac J Cancer Prev, 10, 155-8.

Willems SM, Van Deurzen SHM, Van Diest PJ (2012). Diagnosis of breast lesions: fine- needle aspiration cytology or core needle biopsy? A review. J Clin Pathol, 65, 287-92.

Zidan A, Christie BJ, Peston D, Shousa S (1997). Oestrogen and progesterone receptor assessment in core biopsy specimens 